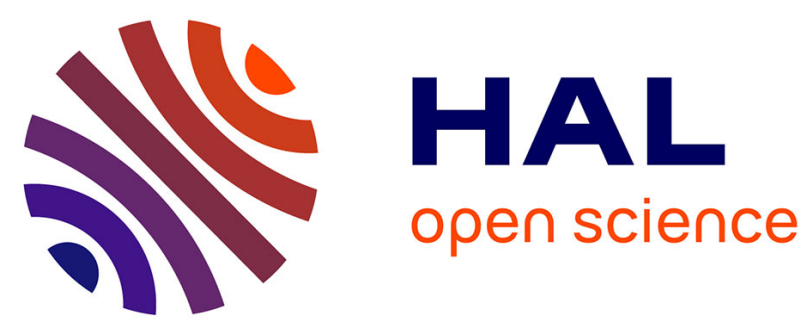

\title{
Status of the CKM Matrix and a simple New Physics scenario \\ Jérôme Charles
}

\section{To cite this version:}

Jérôme Charles. Status of the CKM Matrix and a simple New Physics scenario. Proceedings of the Second Workshop on Theory, Phenomenology and Experiments in Heavy Flavour Physics, Jun 2008, Capri, Italy. pp.17, 10.1016/j.nuclphysbps.2008.10.020 . hal-00327643

\section{HAL Id: hal-00327643 \\ https://hal.science/hal-00327643}

Submitted on 9 Oct 2008

HAL is a multi-disciplinary open access archive for the deposit and dissemination of scientific research documents, whether they are published or not. The documents may come from teaching and research institutions in France or abroad, or from public or private research centers.
L'archive ouverte pluridisciplinaire HAL, est destinée au dépôt et à la diffusion de documents scientifiques de niveau recherche, publiés ou non, émanant des établissements d'enseignement et de recherche français ou étrangers, des laboratoires publics ou privés. 


\section{Status of the CKM Matrix and a simple New Physics scenario}

J. Charles, on behalf of the CKMfitter group ${ }^{\text {a* }}$

${ }^{a}$ Centre de Physique Théorique, Luminy Case 907

13288 Marseille Cedex 9, France

We present a short status of the CKM matrix in the Standard Model as well as possible New Physics contributions to $B \bar{B}$ mixing.

\section{Introduction}

We shall be concerned by the weak current transitions that mix quarks of different flavors, as encoded by the Cabibbo-Kobayashi-Maskawa (CKM) matrix in the Standard Model (SM). The unitarity $3 \times 3$ CKM matrix can be described by four real parameters that we define $\grave{a} l a$ Wolfenstein by

$$
\begin{aligned}
\lambda^{2} & =\frac{\left|V_{u s}\right|^{2}}{\left|V_{u d}\right|^{2}+\left|V_{u s}\right|^{2}} \\
A^{2} \lambda^{4} & =\frac{\left|V_{c b}\right|^{2}}{\left|V_{u d}\right|^{2}+\left|V_{u s}\right|^{2}} \\
\bar{\rho}+i \bar{\eta} & =-\frac{V_{u d} V_{u b}^{*}}{V_{c d} V_{c b}^{*}}
\end{aligned}
$$

This parametrization is exact, unitary and phase convention independent to all orders in $\lambda$. Our aim is to extract as much as possible information from experimental and theoretical inputs so that constraints on the CKM parameters are obtained. Then the questions to be asked follow: does the CKM mechanism describe the experimental data? To which accuracy? Are possible New Physics contributions needed, or simply allowed?

The CKMfitter project follows this program within the Rfit frequentist approach [1]. In these proceedings a few recent results are highlighted.

\footnotetext{
*Unité Mixte de Recherche 6207 du CNRS et des Universités Aix-Marseille 1, Aix-Marseille 2 et Sud ToulonVar, affiliée à la FRUMAM. This work has been supported in part by the EU Contract No. MRTN-CT-2006-035482 'FLAVIANET' and by the ANR Contract ANR-06-JCJC0056 .
}

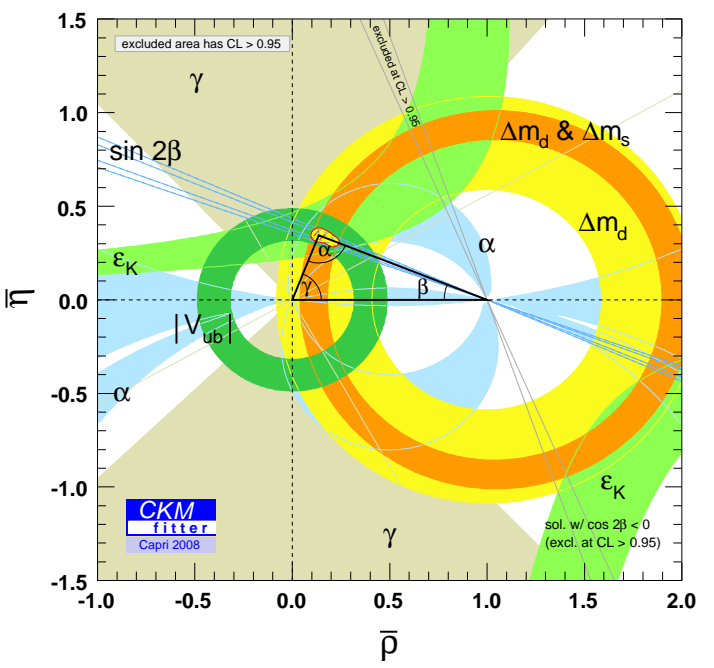

Figure 1. SM constraints at $95 \% \mathrm{CL}$ on the apex $(\bar{\rho}, \bar{\eta})$ of the $B_{d}$ Unitarity Triangle.

\section{The global fit}

The global fit is defined as the combined constraints from the selection of inputs that are both well measured and reasonably well understood from the theoretical point of view. This traditionally corresponds to $\left|V_{u d}\right|,\left|V_{u s}\right|,\left|V_{c b}\right|,\left|V_{u b}\right|$ (from semileptonic decays), $\left|\varepsilon_{K}\right|, \Delta m_{d}, \Delta m_{s}$, $\mathrm{BR}(B \rightarrow \tau \nu)$, and the angles of the Unitarity Triangle $\alpha, \beta$ and $\gamma$, which are the only observ- 
ables that can directly be interpreted in terms of fundamental parameters with negligible theoretical uncertainties.

The detailed list of inputs and references can be found in [1]. There are little changes with respect to the last CKMfitter update done for Winter 2008 conferences [2]. Presumably the most important issues as for the traditional inputs to the global CKM fit are the marginal agreement between the exclusive and inclusive semileptonic determinations of both $\left|V_{c b}\right|$ and $\left|V_{u b}\right|$ [3], and the statistical interpretation of $\gamma[4]$.

The outcome of the global fit is shown in Fig. 1 in the $(\bar{\rho}, \bar{\eta})$ plane, corresponding to the best fit values of the CKM parameters

$$
\begin{aligned}
A & =0.795_{-0.015}^{+0.025} \\
\lambda & =0.2252_{-0.0008}^{+0.0008} \\
\bar{\rho} & =0.135_{-0.016}^{+0.033} \\
\bar{\eta} & =0.345_{-0.018}^{+0.015}
\end{aligned}
$$

\section{New Physics in $B \bar{B}$ mixing}

Even if the global fit within the SM does not show any significant anomaly, it is interesting to study generic New Physics (NP) scenarios in order to quantify better the agreement of the data with the SM predictions, and to find bounds on possible non standard contributions. One of the simplest of these scenarios consists in allowing for arbitrary NP in neutral meson mixing by writing

$$
\begin{aligned}
\left\langle B_{q}\left|\mathcal{H}_{\Delta B=2}^{\mathrm{SM}+\mathrm{NP}}\right| \bar{B}_{q}\right\rangle & \equiv\left\langle B_{q}\left|\mathcal{H}_{\Delta B=2}^{\mathrm{SM}}\right| \bar{B}_{q}\right\rangle \\
& \times\left(\operatorname{Re}\left(\Delta_{q}\right)+i \operatorname{Im}\left(\Delta_{q}\right)\right)
\end{aligned}
$$

where the SM is located at the point $\Delta_{d}=\Delta_{s}=$ 1. Here we will not use observables related to the kaon system.

In order to fit for NP according to the above equation one must first be sure that there is sufficient information to constraint the SM contribution. This is done by assuming that tree-level observables are purely standard, namely $\left|V_{u d}\right|,\left|V_{u s}\right|$, $\left|V_{c b}\right|,\left|V_{u b}\right|, \gamma$ and $\alpha=\pi-\gamma-\phi_{d} / 2$ where $\phi_{d}$ is the 'effective' $\beta$ angle as measured in $B_{d} \rightarrow(c \bar{c}) K_{S}$, that is $\phi_{d}=2 \beta+\operatorname{Arg}\left(\Delta_{d}\right)$. The remaining observables relevant to the global fit depend on both the SM parameters $A, \lambda, \bar{\rho}, \bar{\eta}$ and the NP parameters $\Delta_{d, s}$. Additional useful information on the latter parameters come from the measurement of the semileptonic asymmetries $A_{\mathrm{SL}}^{d, s}$ and the width differences $\Delta \Gamma_{d, s}$ which in our simple NP scenario can be written as

$\Delta \Gamma_{q}=\cos \varphi_{q} \Delta \Gamma_{q}^{\mathrm{SM}}$

where $\varphi_{q}$ is the weak phase $\operatorname{Arg}\left(-M_{12}^{q} / \Gamma_{12}^{q}\right)$. It is important to keep in mind that both the semileptonic asymmetries and the width differences are plagued by significant theoretical uncertainties, which we estimate following Ref. [5].

Recent measurements by the Tevatron experiments have a strong impact on the NP in mixing scenario. Indeed first correlated constraints in the $\left(\phi_{s}, \Delta \Gamma_{s}\right)$ plane from the time-dependent angular analysis of $B_{s} \rightarrow J / \psi \phi$ have been presented by both CDF and D0 [6,7]. These measurements show a discrepancy of about two standard deviations with respect to the SM prediction $\phi_{s}=-2 \beta_{s}=-0.0360 \pm 0.0017$, and have been the basis of a claim for evidence for non standard contributions in $B_{s} \overline{B_{s}}$ mixing in Ref. [8], using a Bayesian treatment of the data.

The interpretation of the Tevatron measurements in view of a combined analysis is not straightforward, for two reasons. The first is that D0, in contrast to CDF, assumes SU(3) flavor symmetry of the strong interaction in order to take as additional input the CP-conserving phases in $B_{d} \rightarrow J / \psi K^{*}$ which in this symmetry limit are identical to the ones in $B_{s} \rightarrow J / \psi \phi$. While this is an interesting approach allowing for a deeper use of the experimental data, it is not known at present to which extent the $\mathrm{SU}(3)$ assumption can be used for final state interaction effects, nor the typical size of the corrections. Ref. [8] attempts to correct for the SU(3) assumption by reintroducing, 'by hand', the mirror solution that is otherwise suppressed by the $B_{d} \rightarrow J / \psi K^{*}$ input. However possible bias and overestimate of the constraint corresponding to the main solution is not taken into account.

Fortunately at the time of the present workshop a new (preliminary) measurement free of the $\mathrm{SU}(3)$ assumption was made public by the D0 collaboration in order to make easier the combination with CDF. This is what we use in the present report. 


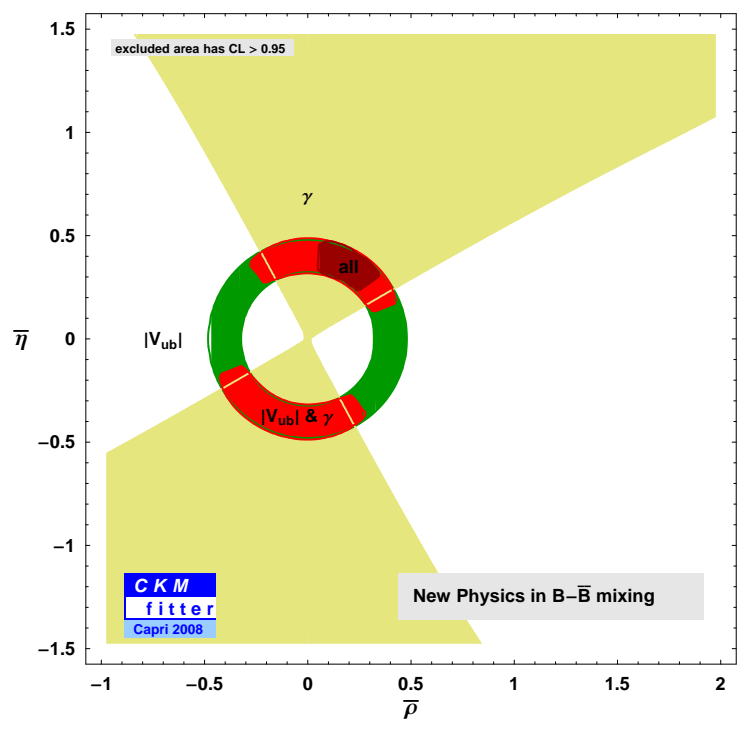

Figure 2. Constraints at 95\%CL on the apex $(\bar{\rho}, \bar{\eta})$ of the $B_{d}$ Unitarity Triangle, within the full $\mathrm{SM}+\mathrm{NP}$ scenario.

The second subtlety in using these data is related to significant preasymptotic (non Gaussian) effects due to the relatively low statistics in both the experiments [6,7]. The naive interpretation of the log-likelihood as a $\chi^{2}$ has the consequence of biasing the fitted estimate of $\Delta \Gamma_{s}$ towards large values, and the fitted error on $\phi_{s}$ towards small values (i.e. the Gaussian assumption is not conservative in this case). This is very much similar to the well known problem of extracting the angle $\gamma$ from asymmetries that are suppressed because of the small value of $r_{B}$. Ref. [6] corrects for the non Gaussianities by using a fully frequentist Monte-Carlo approach, thus constructing Confidence Level contours that have the correct coverage of the true value of the parameters. In the present analysis, though, we use the simplified Gaussian assumption (keeping in mind it is a priori not conservative) because we lack the necessary information from D0 to perform the fully frequentist study. Ref. [8] also uses a similar treatment of the input data.
The results of the full combined analysis within the NP in mixing scenario is shown in Fig. 2 in the $(\bar{\rho}, \bar{\eta})$ plane. It is seen that the resulting allowed region is compatible with the one obtained from the pure SM fit, however it has larger size because of the presence of the additional parameters $\Delta_{d, s}$. The $B_{q} \overline{B_{q}}$ mixing is best explored directly in the $\Delta_{q}$ complex plane, see Figs. 3 and 4 . One

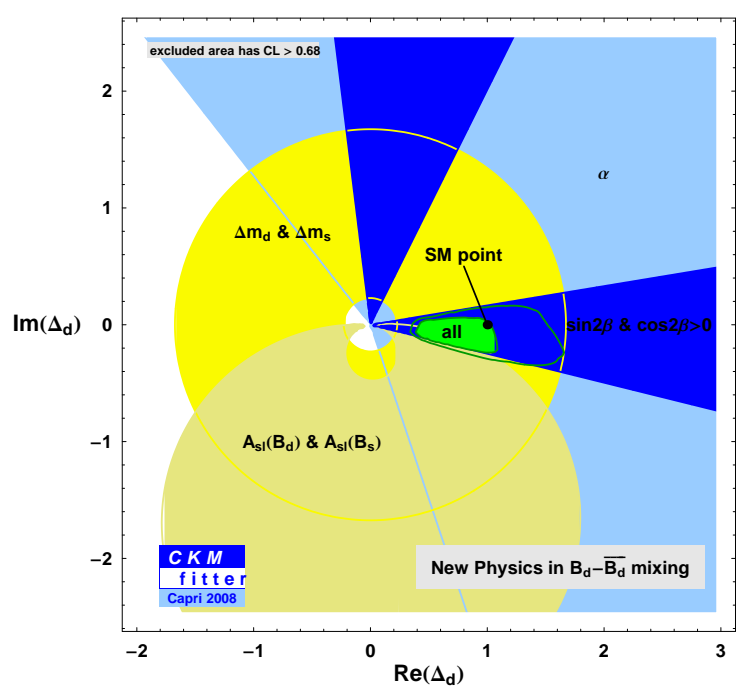

Figure 3. Full SM+NP scenario. Constraints at $68 \% \mathrm{CL}$ on the complex $\Delta_{d}$ parameter, which measures the deviation of $B_{d} \overline{B_{d}}$ mixing with respect to the SM.

finds from these plots that the combined analysis slightly prefers non standard values for the $\Delta_{q}$ parameters, however the effect is not large. In order to put this statement on quantitative grounds, one compute $p$-values for various hypotheses corresponding to the SM predictions, see Table 1 .

The largest $(2.7 \sigma)$ discrepancy with respect to the Standard Model predictions is found when testing (one dimensionally) $\operatorname{Arg}\left(\Delta_{s}\right)=0$ or equivalently $\phi_{s}=-2 \beta_{s}$, which clearly comes from 


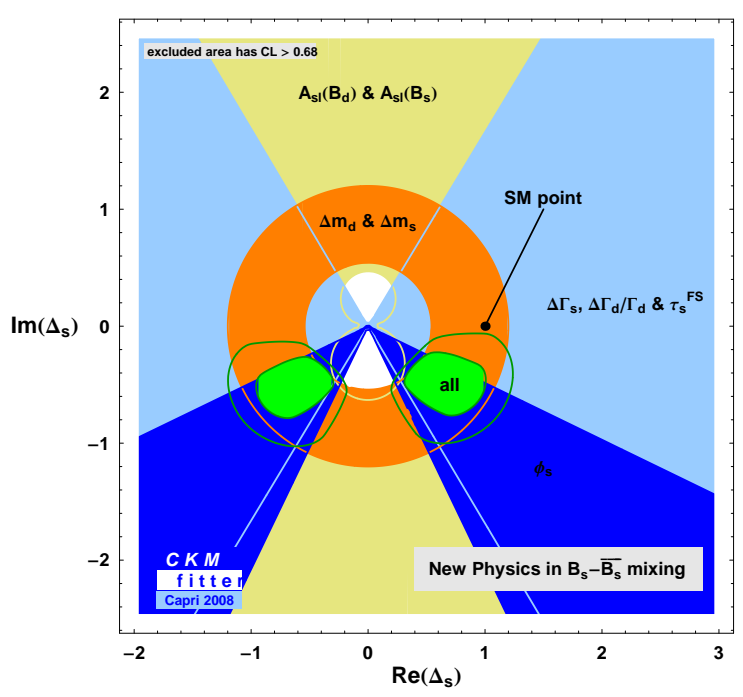

Figure 4. Full SM+NP scenario. Constraints at $68 \% \mathrm{CL}$ on the complex $\Delta_{s}$ parameter, which measures the deviation of $B_{s} \overline{B_{s}}$ mixing with respect to the SM.

the new Tevatron analysis of $B_{s} \rightarrow J \psi \phi$. However the two dimensional hypothesis $\Delta_{s}=1$ is less disfavored, with only 2.2 standard deviations. This is related to the fact that Eq. (4) together with the measurement of $\Delta \Gamma_{s}$ pushes $\varphi_{s}$, and thus $\phi_{s}$ (both phases are equal if the NP phase dominates [5]), towards the SM value. This is best viewed in the $\left(\phi_{s}, \Delta \Gamma_{s}\right)$ plane that we detail below. Indeed one lesson from Fig. 4 is that, as far as $B_{s} \overline{B_{s}}$ mixing is concerned, the only really relevant inputs are $\Delta m_{s}$ and $\phi_{s}$. Since the direct measurement of $\Delta m_{s}$ is well in agreement with the SM prediction, and since $\Delta \Gamma_{s}$ is strongly correlated with $\phi_{s}$, one can perform a much simplified analysis by restricting to the $\left(\phi_{s}, \Delta \Gamma_{s}\right)$ plane and directly comparing with the experimental measurements in [6,7].

Fig. 5 shows the Confidence Level contours in the $\left(\phi_{s}, \Delta \Gamma_{s}\right)$ plane. Again it clearly appears that what drives the minor discrepancy with respect to the $\mathrm{SM}$ is the CP phase $\phi_{s} \cdot p$-values for the SM
Table 1

$p$-values and standard deviations for different hypotheses corresponding to the SM predictions within the generic $\mathrm{SM}+\mathrm{NP}$ scenario

\begin{tabular}{lll}
\hline hypothesis & $p$-value & $\sigma$ \\
\hline $\operatorname{Arg}\left(\Delta_{d}\right)=0$ & 0.37 & 0.9 \\
$\Delta_{d}=1$ & 0.34 & 0.9 \\
$\operatorname{Arg}\left(\Delta_{s}\right)=0$ & 0.0077 & 2.7 \\
$\Delta_{s}=1$ & 0.029 & 2.2 \\
$\Delta_{d}=\Delta_{s}=1$ & 0.07 & 1.8
\end{tabular}

hypotheses corresponding to this simplified plot are reported in Table 2.

Table 2

$p$-values and standard deviations for different hypotheses corresponding to the SM predictions, focusing only on the $\left(\phi_{s}, \Delta \Gamma_{s}\right)$ inputs

\begin{tabular}{lll}
\hline hypothesis & $p$-value & $\sigma$ \\
\hline$\phi_{s}=-2 \beta_{s}$ & & \\
(CDF only) & 0.039 & 2.1 \\
$\phi_{s}=-2 \beta_{s}$ & & \\
$(\mathrm{D} 0$ only) & 0.055 & 1.9 \\
$\phi_{s}=-2 \beta_{s}$ & & \\
$(\mathrm{CDF} \& \mathrm{D} 0)$ & 0.0062 & 2.7 \\
$\phi_{s}=-2 \beta_{s}$ & \\
$(\mathrm{CDF} \& \mathrm{D} 0 \& \mathrm{Eq} .(4))$ & 0.016 & 2.4
\end{tabular}

\section{Conclusion and outlook}

Although there is a hint of a departure from the Standard Model related to the measurement of the phase $\phi_{s}$, consistently by the CDF and D0 experiments, we do not see evidence (larger than the $3 \sigma$ threshold) for New Physics, which has to be contrasted with Ref. [8]. It should be noted that an update of [8] performed for the present workshop leads to a similar conclusion [10]. It thus means that, in addition to possible artefacts of the Bayesian approach, the SU(3) assumption 


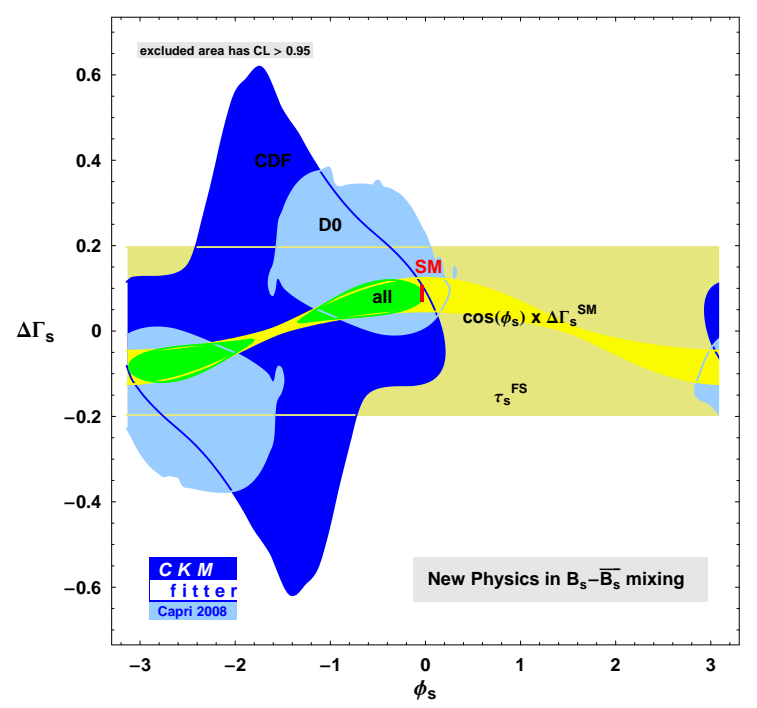

Figure 5. Constraints at $68 \% \mathrm{CL}$ in the $\left(\phi_{s}, \Delta \Gamma_{s}\right)$ plane. Overlaid are the constraints from the direct $\mathrm{CDF}$ measurement, the direct D0 measurement, the constraint from Eq. (4), the constraint from the flavor specific lifetime $\tau_{B_{s}}^{\mathrm{FS}}[9]$ and the overall combination. The SM prediction is also shown.

as originally done in [7] and only partially lifted in [8] significantly increased the discrepancy with respect to the $\mathrm{SM}$ value. In the absence of a better understanding of $\mathrm{SU}(3)$ breaking in non leptonic decays one should not draw definite conclusions about the Standard Model that are based on this assumption.

More importantly we have shown that the bulk of the effect in $B_{s} \overline{B_{s}}$ mixing comes from the direct measurement of $\phi_{s}$ in $B_{s} \rightarrow J \psi \phi$, and that the full combined fit does not really add significant information with respect to the pure experimental analyses in $[6,7]$. This can be clearly understood from the following facts. First, the measurement of $\Delta m_{s}$ is in well agreement with the indirect SM prediction. Second, the semileptonic asymmetries are plagued by too large experimental errors to give a useful constraint in the $B_{s}$ system. And third, the lifetime difference $\Delta \Gamma_{s}$ not only suffers from a significant theoretical uncertainty, but also has a suppressed dependence on the mixing phase in the SM region, because of the cosine function.

Several new measurements that were presented during the Summer 2008 conferences have a significant impact on the analysis presented in this report [11]. This includes the frequentist combination by the HFAG group of the CDF and D0 extraction of $\left(\phi_{s}, \Delta \Gamma_{s}\right)$ [9], which, as expected, relaxes the tension with the $\mathrm{SM}$. On the other hand new data from CDF again go in the NP direction [12]. Finally the new World Average for $\mathrm{BR}(B \rightarrow \tau \nu)$ exhibits a specific correlation with $\Delta m_{d}$ and the CKM angles $\alpha, \beta$ and $\gamma$ that only agrees with the SM at the $2.5 \sigma$ level [11].

\section{REFERENCES}

1. J. Charles et al., CKMfitter group, Eur. Phys. J. C41, 1 (2005); updated results and plots available at: http://ckmfitter.in2p3.fr

2. S. Descotes-Genon, CKMfitter group, talk given at Rencontres de Moriond EW, 1-8 March 2008, La Thuile (Italy)

3. The Review of Particle Physics, C. Amsler et al., Physics Letters B667, 1 (2008)

4. K. Trabelsi, CKMfitter group, talk given at CKM2008, 9-13 September 2008, Roma (Italy)

5. A. Lenz and U. Nierste, JHEP 0706:072 (2007)

6. CDF Collaboration (T. Aaltonen et al.), Phys. Rev. Lett. 100:161802 (2008)

7. D0 Collaboration (V.M. Abazov et al.), arXiv:0802.2255 [hep-ex]

8. M. Bona et al., UTfit collaboration, arXiv:0803.0659 [hep-ph]

9. E. Barberio et al., HFAG group, arXiv:0808.1297 [hep-ex]; online update at http://www.slac.stanford.edu/xorg/hfag

10. L. Silvestrini, UTfit collaboration, these proceedings

11. O. Deschamps, CKMfitter group, talk given at ICHEP08, 29 July-5 August 2008, Philadelphia, PA (United States)

12. D. Tonelli, CDF Collaboration, talk given at ICHEP08 